\title{
LE DYNAMISME ACCENTUEL DANS LE DISCOURS MÉDIATIQUE FRANÇAIS ${ }^{1}$
}

\section{Introduction}

Le présent article a pour but d'exposer une analyse critique sur la nature et le fonctionnement des faits accentuels en français, notamment tels qu'ils sont manifestés dans un corpus sélectionné de textes médiatiques, et surtout ceux qui se présentent dans les émissions d'information télévisées. Ce type de discours présente quelques particularités accentuelles qui sont d'autant plus intéressantes si l'on considère l'influence qu'ont les médias électroniques sur la potentialité de la formation normative d'un code, partagé par les membres (téléspectateurs) d'une communauté linguistique.

\section{L'accent: généralités}

L'accent linguistique est un fait prosodique et l'un des éléments fondamentaux de la compétence linguistique, propre aux locuteurs d'une langue donnée. L'acquisition de la compétence accentuelle a pour résultat l'usage actualisateur dans le processus de la communication: les actualisations de l'accent linguistique - inhérent au système sont automatisées, et leur usage, conditionné par l'apprentissage et par l'expérience communicative, se fait sans interférence de l'effort énonciatif conscient, relatif normalement à l'intentionnalité du message. Évidemment, cela ne signifie pas que le rôle de l'accentuation échappe aux processus conscients qui permettent à l'interlocuteur de reconnaître et de décoder (comprendre) un message linguistique.

Une définition synthétique des faits accentuels pourrait postuler que l'accent est une manifestation prosodique par laquelle, à l'intérieur d'une partie déterminée de la chaîne parlée (unité accentuelle), une unité accentuable (syllabe) est mise en contraste avec toutes les autres syllabes d'une même unité accentuelle. On dit donc que la syllabe marquée par les faits accentuels est accentuée et que les autres sont considérées comme inaccentuées. Il va de soi que toutes les syllabes appelées inaccentuées ne sont pas prosodiquement monotones, mais peuvent au contraire participer à l'image accentuelle générale d'une unité prosodique (ou rythmique). Ces syllabes peuvent se trou-

1 Le texte résume en ses traits principaux la thèse de doctorat que l'auteur a soutenue à la Faculté des lettres de Ljubljana en octobre 1998, devant le jury: prof. dr. Vladimir Pogačnik (directeur de thèse), prof. dr. Janez Orešnik et dr. Rasto Šuštaršič (membres). 
ver affectées par ce qu'on appelle le plus souvent accent secondaire ${ }^{2}$ ou par les différentes manifestations fonctionnelles et substantielles d'un autre type d'accent, dénommé communément accent d'insistance.

Dans toutes ses manifestations dans les différentes langues, l'accent exerce la fonction de marqueur prosodique et de porteur de sens (mais pas toujours de signification ${ }^{3}$ ) à l'unité accentuelle énoncée. Ce fonctionnement de base génère dans la variation des codes linguistiques au moins deux types évidents de langues: relativement à la prévisibilité de la position de l'accent, on distingue entre les langues à accent fixe et celles à accent mobile. Il faut toutefois ajouter que la fonction contrastive linguistique de l'accent dans les deux cas ne varie pas fondamentalement. Dans certains codes linguistiques (notamment dans les langues à accent fixe), les fonctions de délimitation et de contraste entre les unités accentuelles (et de sens) peuvent être assurées par la variation des formes intonatives. On verra plus tard que l'accent et l'intonation de la phrase, dans certains cas, peuvent affecter et déterminer simultanément la même partie de la chaîne parlée; cela ne veut pourtant pas dire que - du point de vue de la cohérence communicative du message linguistique - la fonction de l'un ou de l'autre fait prosodique en question soit redondante.

\subsection{Substance de l'accent}

La syllabe, qui se définit comme le domaine d'opération des faits accentuels, est une unité dite accentuable de la structure représentative de la chaîne parlée et comme telle immédiatement supérieure à celle du phonème. Dans la classification systémique des formes linguistiques, et selon son inopérativité au niveau segmental, l'accent sera donc considéré comme un fait suprasegmental ou, en termes fonctionnels, prosodème. ${ }^{4}$

2 P. Garde (1968), p. 53: «L'existence d'un contraste entre la syllabe accentuée et les syllabes non accentuées ne signifie pas que ces dernières soient homogènes. Dans la plupart des langues à accent les syllabes inaccentuées sont inégales entre elles, et la répartition de l'intensité (ou des autres procédés accentuels) entre elles est réglée par leur position par rapport à l'accent. Ainsi la configuration accentuelle de l'ensemble du mot, avec le plus ou moins de relief de chacune des syllabes, apparait-elle comme un corollaire des autres procédés accentuels, une forme de réalisation de l'accent. On dit généralement de celles des syllabes inaccentuées qui reçoivent la plus grande part des facteurs accentuels qu'elles portent un accent secondaire. Ce terme étant ambigu, nous dirons ici qu'elles reçoivent un écho de l'accent.».

3 Le cadre théorique de cette analyse distingue nettement entre la signification d'une partie donnée de la chaîne parlée et son sens. La signification en tant que qualité sémantique du lexème est une valeur linguistique représentative qui se trouve transposée au sens lors de l'actualisation (à travers l'acte de parole) en un énoncé. Le sens est donc donnée par la contextualisation de la (des) signification(s) et par l'interaction dans la situation communicative du locuteur et de l'interlocuteur.

4 Le prosodème est une unité prosodique fonctionnelle. Il affecte donc certaines parties de la chaîne parlée, dont l'extension est supérieure à celle du phonème ( $p$. ex. syllabe, mot, syntagme, phrase). Son fonctionnement est assuré substantiellement par l'intensité, la variation tonale ou par la durée (ou par les diverses combinaisons de ces composantes acoustiques) qui déterminent l'image prosodique d'une phrase ou de ses parties. La fonction du prosodème ne peut pas se déterminer uniquement par ses propriétés physiques inhérentes. Il est toujours actif dans les relations de cohérence avec les autres niveaux d'énonciation. Son fonctionnement se définit donc par l'interaction dans laquelle entre le prosodème par rapport aux autres éléments de son contexte phonique. 
La réalisation de différents types d'accent peut engager tous les trois paramètres prosodiques principaux de la formation des chaînes parlées: l'intensité, la hauteur et la durée. Pour la plupart des langues - dont le français - on a l'habitude de dire que la syllabe accentuée est réalisée par une valeur relativement accrue de l'intensité vocalique à laquelle s'ajoute le plus souvent une durée plus longue. C'est pourquoi la tradition linguistique a tendance d'appeler cet accent par le critère substantiel, donc accent d'intensité. Il est plus précis de dire que la réalisation de chaque type d'accent est le résultat d'un effort expiratoire ${ }^{5}$ particulier et que l'impression auditive de l'accent est une projection acoustique complexe de cet effort sur la syllabe affectée. On dira donc que la substance de l'accent est une combinaison de facteurs prosodiques dont l'un ou l'autre peut être proéminent dans certains types d'accent.

\subsection{Fonctions de l'accent}

En termes fonctionnels, l'accent exerce d'abord le rôle de mise en relief de la syllabe accentuée, rôle relatif à sa valeur culminative. Cette fonction a pour but de distinguer la réalisation d'un sommet physique de l'unité accentuelle par rapport à son reste «inaccentué»).

La seconde fonction que devrait remplir l'accent linguistique s'effectuerait par l'activation de sa valeur démarcative ou délimitative: en français, l'accent est censé indiquer la fin du mot phonétique, puisqu'il paraît frapper régulièrement sa dernière syllabe. Ici, on essaiera cependant de montrer quelles sont, dans la réalisation de la chaîne parlée, les conséquences de cette fixité prétendue de l'accent français. ${ }^{6} \mathrm{Il}$ paraît justifié d'avancer que la fixité absolue de n'importe quel fait linguistique (et plus encore, prosodique) détermine la réalisation de ce même fait jusqu'à ce qu'elle devient généralement prédictible et, étant donné son automatisme, relève du choix inconscient du locuteur.

L'examen fonctionnel général des diverses manifestations de l'accentuation ${ }^{7}$ attribue à l'accent une troisième fonction, notamment celle qui découle de sa puissance distinctive. Or il est important de préciser tout de suite que la distinctivité de l'accent ne saurait être réellement associée à la valeur distinctive qui, selon les principes de pertinence phonologique, est inhérente aux propriétés essentielles du phonème. Fonctionnant dans la chaîne parlée au niveau contrastif, l'accent peut être distinctif dans les langues où il est également considéré comme mobile ou «non-fixe», comme en slovène, en allemand, en serbe, ou, d'ailleurs, dans la plupart des langues romanes. Dans ces

5 I. Fónagy (1983) avance dans son étude «psychophonétique» que l'accentuation résulte d'une activité physiologique momentanément intensifiée de la musculature toracale et abdominale.

6 If semble utile de signaler en passant que ce genre d'idéalisations, souvent provenant d'une attitude non-critique propre à l'enseignement grammatical «latin», est adapté aux besoins didactiques qui sont à la base du transfert des connaissances linguistiques générales à une grande plupart des niveaux scolaires.

7 Naturellement, ce texte ne se propose pas de traiter de diverses manifestations d'accent, réalisé au moyen de la variation tonale opérant, selon le principe de substitution, sur le développement d'une seule voyelle, c'està-dire de l'accent tonématique qui figure sous forme phonologique de tonème. 
cas-là il peut, à la rigueur, former la base de construction de paires minimales contrastives. Toujours est-il qu'il opère ses contrastes non pas sur le principe de substitution formelle, mais - pour ainsi dire - selon la règle d'《être ou ne pas être». La distinctivité de l'accent n'entrera donc pas en jeu quand on tentera de décrire le fonctionnement de l'accent final en français, puisqu'il n'est guère en position, grâce aussi à sa fixité présumée, d'apporter une possibilité de distinction entre deux sens différents, et encore moins de distinguer des significations.

Comme il a déjà été indiqué, l'accent n'a pas de statut phonologique proprement dit; il n'est donc pas doué de valeur de pertinence que pourrait lui attribuer la phonologie structurale linéaire. Celle-ci ne prend en considération que les manifestations phonétiques que l'on peut, conformément aux résultats qu'elles donnent dans une chaîne parlée, systémiser selon leur fonctionnement sur l'axe paradigmatique. Cela signifie qu'un phonème ou un de ses traits articulatoires ou acoustiques (audio-perceptifs) peut être substitué ou commuté par un autre élément du même ordre phonologique, recevant ainsi une valeur distinctive phonologique, et formant la base de construction de paires minimales (p. ex.: rat - chat). La nature même de la manifestation accentuelle (hormis les tonèmes) est telle qu'une syllabe déterminée (accentuable) porte ou ne porte pas l'accent. Cela fait de l'accent linguistique - qu'il soit fixe ou non-fixe - un phénomène prosodique qui exerce ses fonctions sur l'axe horizontal de la chaîne parlée et qui, par conséquent, ne peut avoir qu'une valeur contrastive. Le fonctionnement de l'accent paraît particulièrement limité en français où la possibilité de mettre en contraste les syllabes est diminuée par la position immuable de l'accent. Il semble en plus que cette propriété entraîne une certaine supposition de diminution du rendement démarcatif. Répétons que cette position fixe et absolument prévisible, position donc propre à la construction de l'unité accentuelle française, peut même avoir une conséquence concernant le fonctionnement de diverses structures énonciatives: à savoir, ce type de positionnement de l'accent linguistique sur la dernière syllabe de l'unité correspond précisément à l'emplacement de la conclusion intonative. Autrement dit, l'accent affecte régulièrement et prévisiblement la syllabe sur laquelle se situe l'événement intonatif, déterminant la forme intonative qui se réalise sur une unité accentuelle donnée.

\subsection{L'unité accentuelle}

L'unité accentuelle se définit comme une partie de la chaîne parlée, réalisée lors de l'énonciation en une seule émission de souffle (unité de souffle), et qui comporte une seule syllabe affectée par l'accent linguistique à valeur délimitative ou distinctive (au niveau contrastif). Selon l'aspect prosodique et en co-opération de l'accentuation avec l'intonation phrastique - ce qui est surtout valable pour les langues à accent fixe - l'unité accentuelle constitue également une unité de rythme. Etant donné que, dans le processus de l'actualisation, une unité rythmique est douée de valeur sémantique complexe, l'unité accentuelle est au même titre considérée comme une entité de sens. 
D'entre les unités linguistiques formelles il y en a deux qui, en tant que porteuses de signification et créatrices du sens, sont les plus autonomes. Le morphème est l'unité significative minimale qui, du point de vue de sa valeur sémantique, est inanalysable en unités de structure superficielle inférieures. Dans cette même optique, la phrase est l'unité de sens extensive qui n'a pas besoin d'être complétée sémantiquement dans le processus de l'actualisation et qui est conçue par les participants à la communication comme un organisme significatif. Le statut du mot doit être recherché, selon $\mathrm{Garde}^{8}$, entre ces deux concepts. Aussi est-il vrai que les manifestations linguistiques mono-morphémiques peuvent se représenter sous forme d'un seul mot, et qu'une phrase peut être construite d'un mot seulement (p. ex. «alors» ou «Applaudissez!»), mais en général, ce que l'on considère comme mot contient normalement plus d'un morphème et ce qu'on appelle phrase englobe le plus souvent plusieurs mots. L'incompatibilité formelle du «mot» avec le concept de «morphème» et celui de «phrase» nous amène à constater que la définition du mot par les moyens grammaticaux devrait être substituée par celle qui a recours à la formalisation sémantique («lexème») ou par celle qui se sert de critères phonétiques ou prosodiques. Autrement dit, il est justifié de déterminer le «mot» phonologique ou prosodique comme l'une des formes possibles de ce que l'on appelle unité accentuelle.

\section{La notion de l'accentuation en français contemporain}

Selon l'acception habituelle de l'accentuation en français, les manifestations accentuelles sont divisées en deux sous-types, les deux fonctionnels, mais dont l'un représente une partie élémentaire de la structure inhérente du code linguistique (accent final), tandis que l'autre est réalisé dans le cadre des stratégies énonciatives individuelles, résultant du choix intentionnel du locuteur (accent d'insistance).

\subsection{L'accent final}

L'histoire de la description de l'accent final en français est longue et hétérogène. Les innombrables variantes des dénominations ${ }^{9}$ de cet accent inhérent au code lingui-

8 P. Garde (1968), p. 18: «Si la notion de mot se confondait soit avec celle de morphème, soit avec celle de phrase, elle serait superflue. Et de fait la notion de mot est inutile dans les langues où on l'emploie pour désigner les morphèmes: c'est le cas des langues «monosyllabiques», comme le thaï, qui sont justement des langues sans accent. Mais dans la plupart des langues la notion de mot se justifie, parce qu'elle désigne précisément une unité intermédiaire entre le morphème et la phrase.».

9

Les plus anciennes descriptions de l'accent final français sont souvent issues de la conception de la grammaire latine. Ainsi, cet accent s'est vu attribuer des adjectifs techniques comme «historique», «étymologique» et même des termes comme «ictus roi» ou «ictus rex». La fixité de l'accent final est à l'origine du terme traditionnel (mais peu précis) «accent normal». D'un autre côté, dans leurs efforts terminologiques les linguistes ont eu également recours à la substance de l'accent final: on l'appelait donc "accent d'intensité» quand on voulait souligner le rôle acoustique de l'intensité vocalique ou «accent tonique» quand, à l'instar des néo-grammairiens, on désirait le définir comme l'élément proéminent par rapport aux syllabes inaccentués (dans cette 
stique français tiennent leur origine dans l'incertitude des linguistes par rapport aux critères de définition. L'accent final, comme d'ailleurs tous les autres types d'accent, est un fait prosodique complexe dont l'image acoustique est le résultat d'une activité physiologique par laquelle le locuteur met la syllabe accentuée en contraste avec les autres syllabes de la même unité accentuelle. Cette caractéristique essentielle de l'accent a généré une dénomination généraliste mais précise qui tient compte de tous les facteurs qui sont linguistiquement pertinents à la description de ce fait: on appelle donc l'accent final français, en un premier lieu, accent dynamique. ${ }^{10}$

Du point de vue de son origine substantielle, l'accent final est considéré comme accent d'énergie ou accent expiratoire. Dans le bref aperçu des généralités accentuelles, on a déjà montré l'insuffisance d'une décision arbitraire pour l'un ou les autres parmi les différents paramètres acoustiques qui, en fait, se combinent tous dans la matérialisation sonore de l'effort expiratoire.

La fonction des faits linguistiques constitue souvent le critère descriptif qui facilite considérablement le problème de leur étiquetage notionnel. La position finale de l'accent français (et avec cela le classement du français dans le type des langues à accent fixe) a pour résultat incontestable la transparence présumée de sa fonction. C'est par le recours automatisé à l'emploi de l'accent final que le locuteur français délimite entre elles les unités accentuelles; d'un autre côté, c'est de ce même moyen accentuel que se sert l'interlocuteur afin de s'orienter dans la complexité du sens de l'énoncé. Il est donc justifié de dire que sa fonction qualifie l'accent final français en tant qu'accent délimitatif ou orientatif. Reste, bien sûr, à voir quelles sont les conséquences de l'évidente interaction dans laquelle entrent l'accent final et l'événement intonatif dans l'acte de parole, produit par un locuteur se servant du code français.

\subsection{L'accent d'insistance}

Le second type d'accent français, normalement, ne fait pas l'objet des analyses liguistiques oppositionnelles, puisqu'il s'agit d'un fait prosodique qui n'entre pas dans la structure inhérente du code français. L'usage de l'accent d'insistance ${ }^{l l}$ est donc le résultat d'un choix individuel que le locuteur fait en fonction de ses stratégies énonciatives et communicatives.

même optique terminologique on distingue entre les pronoms «atones» et «toniques»). Il va sans dire que la terminologie linguistique contemporaine désigne du qualificatif «tonique» la propriété d'un certain type d'accent dont la substance est une variation microprosodique de la hauteur tonale sur une seule voyelle et dont la forme est couramment appelée «tonème».

${ }^{10} \mathrm{C}$ 'est peut-être la seule définition terminologique de l'accent final français qui relève en même temps de la nature de sa substance et de sa fonction. En plus, ce terme explique le dynamisme évolutif de son comportement dans la chaîne parlée française.

11 L'accent d'insistance servant à exprimer certaines prises de position, provenant de l'individualité du locuteur par rapport au contenu de l'énoncé, la dénomination de ce type prosodique ne serait pas moins claire si l'on l'appelait «accent d'attitude». 
Il est éventuellement possible de résumer les propriétés fonctionnelles et substantielles en trois facteurs formels:

1. L'accent d'insistance est fondamentalement un élément individuel du schéma prosodique d'une unité accentuelle; son actualisation dépend donc de telle ou autre attitude (émotive ou logique, explicative) que le locuteur exprime selon l'intention communicative de son message linguistique.

2. Le champ d'opération de l'accent d'insistance, par définition, est le lexème. Par l'emploi de cet accent, le locuteur valorise intentionnellement l'unité significative choisie selon sa stratégie énonciative.

3. L'accent d'insistance se place normalement sur la première syllabe du lexème; cette position forme évidemment le contraste spatio-temporel avec l'accent inhérent qui affecte la dernière syllabe de l'unité accentuelle.

Nous verrons plus tard que les conséquences de l'emploi régularisé de l'accent d'insistance dans le discours médiatique français modifient sensiblement la deuxième constatation, relative à l'unité de la chaîne parlée, marquée par ce trait prosodique.

\subsection{Virtualité de l'unité accentuelle en français}

La délimitation accentuelle des unités de sens dans le processus de l'énonciation dépend fortement du débit et de la stylisation de la parole. La phrase Pierre va venir à la maison vers dix heures du soir, actualisée en débit rapide, peut n'être divisée rythmiquement qu'en deux unités: Pierre va venir à la maison / vers dix heures du soir. Il est également possible d'imaginer que la même phrase pourra se réaliser en trois (Pierre I va venir à la maison / vers dix heures du soir), en quatre (Pierre / va venir à la maison / vers dix heures / du soir), voire en cinq (Pierre / va venir / à la maison / vers dix heures / du soir) unités accentuelles. Toutefois, la phrase qui nous a servi d'exemple, ne peut aucunement comporter plus de cinq accents délimitatifs, nombre donc qui correspond à l'organisation syntaxique de la phrase en constituants immédiats du discours. Il est de loin plus probable qu'elle en comportera moins de cinq, et cela dans la grande plupart des actualisations possibles. Le nombre de pauses et d'accents décroît avec la rapidité du débit. Ayant pris conscience de cette caractéristique essentielle de l'accentuation finale en français, nous sommes amenés clairement à ne pas égaliser absolument l'unité accentuelle à l'unité syntaxique (ou au constituant immédiat); il est beaucoup plus précis de parler des unités accentuelles françaises en termes d'unités possibles ou potentielles. La terminologie de Paul Garde attribue à l'unité accentuelle en français la qualité de virtualité: «[...] en français, l'unité accentuelle effectivement réalisée dans la parole a des limites très variables, qui dépend de la succession des syllabes susceptibles d'être accentuées, du rythme de la parole et des pauses, et il n'existe pas, dans la langue, un seul mot qui ne soit susceptible, dans certaines positions de perdre son accent. L'accent affecte en fait non pas des unités dont on puisse don- 
ner une définition grammaticale permanente, mais des unités dont les limites varient d'un énoncé à l'autre.»12

C'est par là, entre autres moyens de contraste acoustique, que la prosodie française évite le contact direct de deux accents, contact qui serait nuisible à l'intelligibilité du sens du message linguistique. Il est donc probable dans l'acte énonciatif qu'un lexème directement antéposé à une unité accentuelle monosyllabique perdra son accent. Dans les phrases ou syntagmes, tels que une feuille morte, il parle mal ou ça ne va pas, un seul accent est perceptible, notamment celui du mot monosyllabique final. Cette tendance d'éloignement des accents dans la chaîne parlée française peut aussi avoir pour résultat la modification de la position accentuelle dans le lexème antéposé au monosyllabique accentué. Le syntagme Sacré-Coeur, dans l'actualisation orale, sera donc accentué ['sakre'kœR] ou [sakre'kœR], mais jamais en variante [sa'kre'kœR] qui paraîtrait correspondre aux règles de l'accentuation finale.

La structuration morphémique et syntaxique de l'énoncé français permettent donc uniquement de déterminer d'éventuelles unités accentuelles minimales (virtuelles); ce sont les unités qui comportent une syllabe accentuable. Il n'est donc pas plus possible de définir l'unité accentuelle en critères de syntaxe que de décrire l'unité accentuable en termes morphosyntaxiques stricto sensu.

Les règles normatives de l'accentuation en français posent en même temps un schématisme dans lequel toutes les proclytiques (notamment les articles, indices pronominaux, indices déictiques, etc.; autrement dit: les mots grammaticaux) sont proclamées inaccentuables de façon à ce qu'elles ne peuvent pas constituer le noyau accentuel de l'unité rythmique. On estime traditionnellement que les proclytiques sont incluses dans des unités accentuelles minimales ou plus étendues. Celles-ci, à leur tour, ne sont pas toutes réalisées, et doivent donc être considérées en termes de virtualité.

\subsection{La substance de l'accent délimitatif français}

Fónagy ${ }^{13}$ constate que les propriétés dynamiques de la substance accentuelle (acoustique) deviennent plus transparentes si l'on considère le développement historique de l'accentuation du français et de son évolution phonétique en général: «Nous sommes amenés dans l'analyse de l'histoire du consonantisme et du vocalisme à distinguer entre les sons qui se trouvent en syllabes accentuées et ceux qui sont en syllabes atones. La centralisation des voyelles en syllabes atones, leur chute en finale absolue, l'allongement de la voyelle ou la diphtongaison (montante) en syllabe accentuée s'expliquent et ne s'expliquent que par l'effort accentuel.»

Il faut souligner ici que les résultats du processus articulatoire (expiratoire) de la production accentuelle sont pour le locuteur essentiellement différents des effets pro-

12 Garde (1968), p. 94.

13 Fónagy (1979), p. 128.

106 
sodiques provenant des procédés qui permettent au participant à la communication de percevoir, de reconnaître et d'évaluer les accents ou les schémas accentuels. Les diverses analyses perceptives ${ }^{14}$ de l'accent français affirment que la reconnaissance des syllabes accentuées n'est pas exclusivement conditionnée par la perception des valeurs acoustiques proéminentes. L'examen des processus perceptifs démontre que les diverses structures linguistiques codifiées (entre elles l'accent et l'intonation phrastique) ne sont pas reconnues normalement en tant que structures énonciatives indépendantes (et encore moins auto-suffisantes). On les perçoit au contraire comme des procédés énonciatifs parallèles aux autres structures linguistiques (syntaxe), formant ensemble la cohérence communicative du message.

Le fait qu'une unité accentuelle actualisée en français correspond - le plus souvent - exactement à l'extension d'une unité intonative sous-tend l'hypothèse de l'interaction acoustique de différents facteurs prosodiques sur une même syllabe. Si l'accent final frappe régulièrement la dernière syllabe de l'unité, c'est en même temps la syllabe sur laquelle s'opère l'événement intonatif. Il faut donc constater que le dynamisme intonatif affecte la syllabe accentuée par le changement de la hauteur tonale et que l'événement intonatif doit être considéré comme une action simultanée par rapport à celle de l'accent. Du point de vue de la substance cela représente donc une nouvelle confirmation de l'opinion de Fónagy (complexité de la projection acoustique sur la syllabe accentuée); cependant, l'explication de l'aspect fonctionnel de la syllabe finale française demande une précision importante. Etant donné l'interaction des deux faits prosodiques sur la même position, la fonction délimitative de l'accent se trouve à chaque fois nettement complétée par le fonctionnement simultané de l'action intonative. Il y a donc deux prosodèmes à la fin de l'unité accentuelle (et intonative) française qui assurent la délimitation des parties de la chaîne parlée et l'orientation dans le sens de l'énoncé.

\section{Corpus}

Le corpus de textes parlées, enregistrés sur plusieurs chaînes de télévision françaises, belges, suisses et canadiennes, comporte environ 10500 mots analysés. On a porté le plus d'attention sur la parole médiatique, telle qu'elle est produite par les animateurs (VOD), journalistes (NOV) et invités (INT) se présentant dans les journaux télévisés. L'analyse a inclus une émission de débat culturel avec, également, la participation d'un animateur et de quelques invités.

La recherche se fonde sur la supposition que les animateurs et les journalistes de diverses provenances francophones utilisent professionnellement la langue française en tant qu'instrument d'un discours normatif, approprié aux intentions communica-

14 Entre autres Rossi $(1979,1981)$ et Carton (1974). 
tives des messages médiatiques. ${ }^{15}$ Une deuxième prémisse analytique est celle de l'influence de ce type de discours sur les réalisations des invités, des interviewés, bref, des participants médiatiquement non-professionnels aux émissions étudiées. L'exposition de ce groupe de locuteurs aux particularités énonciatives (prosodiques) des animateurs et des journalistes se trouve rejointe par la spécificité de la «situation communicative médiatique», donc par l'emplacement de la communication (studio) et par la présence des caméras et des micros.

Dans la notation «orthographique» du corpus, les limites des unités accentuelles (intonatives) sont posées par les italiques qui représentent les accents délimitatifs. Tous les autres faits accentuels se plaçant ailleurs qu'en fin de l'unité (y compris les accents d'insistance) sont marqués en caractères gras. La barre oblique $(/)$ marque les pauses qui séparent les unités accentuelles se terminant normalement par l'italique ou encore par une virgule. Deux barres obliques désignent une pause plus longue.

\subsection{Un extrait du corpus}

\section{Journal France $3 \quad[27 / 41998,12.30]$}

(VOD-1) En Allemagne, le parti du chancelier Helmut Kohl, vient de subir une nouvelle défaite, tandis que celui de son adversaire, Gerhard Schroeder, poursuit sa progression. A cinq mois des élections fédérales, ce scrutin régional / de Sachse Anhalt, est un avertissement pour le chancelier, mais ce scrutin / marque aussi surtout la montée / des communistes / et des néo-nazis dans cette région sinistrée de l'ex-RDA. JeanPaul Gérouard.

(NOV-1) Dimanche noir, catastrophe, signal d'alarme, ce matin, les journaux allemands rivalisent de titres choc. Le résultat des élections dans le lander de Sachse Anhalt est effectivement un véritable coup de tonnerre politique. Le parti chrétien démocrate d'Helmut Kohl perd douze points, la gauche progresse moins que prévu, mais surtout l'extrême droite fait une percée totalement inattendue. Avec / douze virgule neuf pourcent des voix, le parti néo-nazi, le DVU, fait son entrée dans le parlement de lander de l'ancienne Allemagne de l'Est pour la première fois. Dirigé depuis Munich par un milliardaire, il a mené une campagne / ultra xénophobe / avec de gros moyens financiers, on ne l'attendait tout de même pas à un tel niveau, puisque le lander de Sachse Anhalt / compte moins de deux pourcent d'immigrés. Aujourd'hui, les

\footnotetext{
${ }^{15}$ L'intention la plus générale du discours médiatique est l'information du public. Cette intention est censée orienter l'opinion publique et le comportement du spectateur. Dans le sens linguistique il s'agit de comprendre l'information en tant que confrontation du spectateur avec la codification médiatique du discours, l'orientation en tant qu' influence sur la formation de la compétence linguistique du spectateur, et le comportement en tant qu' influence d'un discours particulier sur les performances linguistiques eventuellement modifiées par le code médiatique.
} 
analystes s'accordent pour expliquer cette percée par le malaise et la précarisation sociale dans l'ex-RDA, le taux de chômage / en Sachse Anhalt / atteint désormais / vingt-deux virgule six pourcent. Cette élection constitue de toutes façons un nouveau fiasco pour Helmut Kohl, ce matin, la presse s'interroge sur sa capacité à mener son parti pour les prochaines élections législatives, dans cinq mois.

(VOD-2) L'assassinat d'un / défenseur des droits de l'homme au Guatémala, il s'agit de l'évêque / Juan Gerardi qui a été / tué / la nuit dernière.

(VOD-3) Oui je viens / de / l'apprendre, l'ancien sénateur varois et ancien maire de Toulon, Maurice Arex, a été interpelé / ce matin/à Toulon, pour purger un seul de huit mois de prison / qu'il / doit effectuer à la maison d'arrêt des Baumetz.

(VOD-4) Un mois / après l'arrestation du tueur en série de l'Est parisien Guy Georges, l'instruction se trouve / totalement paralysée. Au total, trois juges / instruisent / les dossiers, aucun d'entre eux/ ne veut se désaisir, les familles des victimes sont véritablement écoeurées par cette bataille qui dessert la recherche de la vérité, car le meurtrier présumé / refuse d'être entendu / par / les trois magistrats.

(VOD-5) A présent, on découvre / pour la première fois / le billet / qui nous ouvrira / les portes / de la Coupe du monde de football, Michel Platini l'a présenté à la presse ce matin, comme l'affiche, ce billet est plutôt coloré, Didier Balaise.

(MÉTÉO) Eh bien pas d'amélioration en vue, on peut dire que la perturbation, qui traverse la France depuis trois jours, va encore persister sur l'est du pays, elle donne des pluies considérables, MétéoFrance dit même qu'on risque des crues dans le Massif central, toutes les régions du Cantal, du centre, avec des précipitations très importantes // depuis plusieurs jours donc prudence // nous avons encore un petit peu de neige sur les Alpes, les Pyrénées et surtout un caractère très orageux / sur la moitié sud du pays où là, on attend encore des pluies, donc un caractère orageux, heu, très / conséquent aujourd'hui, puisque les // effectivement, les précipitations sont très nombreuses depuis plusieurs jours.

Journal France Télévision $2 / 3 \quad$ [27/4 1998, 22.00 ]

(VOD-11) La vente des billets pour la Coupe du monde de football, suite à quarantequatre jours du coup d'envoi, le stock diminue lentement sur les cent dix mille billets, offerts au public européen, mercredi dernier, il en reste à peu près vingt-cinq mille en vente par téléphone, mais tous maintenant pratiquement pour des matchs du 
tour préliminaire. Au fait, savez-vous à quoi ressemble le billet, savez-vous qu'il est infalsifiable? Didier Balaise.

(NOV-18) Voici l'objet de toutes les convoitises, le précieux Sésame qui ouvrira la porte pour assister à l'un des soixante-quatre matchs de la Coupe du monde. Deux millions et demi de billets seront émis avec pour priorité, la lisibilité, l'esthétique et bien sûr, la sécurité, résultat, ce billet est presque / infalsifiable.

(INT-11) Oui je crois qu'il est vraiment infalsifiable // vraiment infalsifiable pourquoi, parce que ses sécurités sont dans le papier lui-même. C'est-à-dire que non seulement il est impossible d'en faire simplement une photocopie, mais surtout il est même impossible à un imprimeur de le reproduire.

(NOV-19) Pour éviter la fraude ou les falsifications, hologramme, filigrane, numéro du client et nom de l'acheteur seront facilement identifiables. Reste que le commité d'organisation s'est fait beaucoup d'ennemis: sur trente millions de demandes seulement deux millions et demi ont été satisfaites, grâce à une politique de prix très raisonnables.

(INT-12) Je crois qu'on a fait ce qu'il fallait faire // dans la clarté de la transparence depuis quatre ans //mais le gens ne sont pas contents // parce qu'il manque des billets // et bah tant pis. La prochaine fois on demandera à l'état français de faire des stades de cent mille, on va les remplir pour quatre matchs, et puis après, ils seront vides pour cinquante ans.

(NOV-20) Mais avant cela, quarante mille billets supplémentaires, retournés par certaines petites fédérations, seront remis à la vente / dès cette semaine. Les plus chanceux devraient recevoir le prestigieux ticket / par la poste dès la mi-mai.

Journal R TBF $\quad[28 / 41998,01.00]$

(VOD-1) Dix morts et trente-sept blessés, voilà le / bilan d'un accident de car, qui s'est produit hier / dans le sud-est / de l'Espagne, à Villa Franquesa dans la province d'Alicante, un accident meurtrier donc, et aussi très spectaculaire, le véhicule est passé au-dessus d'un pont et s'est écrasé vingt-cinq mètres plus bas, le car transportait des retraités espagnols.

(VOD-2) «Les nazis / dehors », c'est avec ce slogan qu'une trentaine de personnes ont violemment / protesté hier soir en Allemagne, contre le score obtenu par un parti d'extrême droite. Lors de l'élection régionale dans un land de l'ancienne Allemagne de l'est, l'Union du peuple allemand, y a en effet obtenu treize pourcent des voix. Philippe Lemaire. 
(NOV-1) Aux élections régionales de Sachse Anhalt, c'est une énorme surprise qui est sortie des urnes. Outre la défaite puisante du parti d'Helmut Kohl, la $\mathrm{CD} U$, qui perd un tiers des voix par rapport au scrutin de mille-neuf-cent nonante-quatre, c'est surtout l'entrée de l'extrême droite dans l'assemblée régionale de ce land qui a sonné comme un coup de tonnerre dans le ciel politique allemand. La DVU, l'Union du peuple allemand, sposorisée par un milliardaire munichois, remporte en effet d'emblée près de treize pourcent des suffrages. Dans une région minée / par le chômage et la crise économique, le discours démagogique et raciste de l'extrême droite a payé. C'est la première fois qu'un parti d'extrême droite / entre dans un parlement régional de l'ex-/ $\mathrm{RD} A$, depuis la réunification / allemande. Outre les socio-démocrates, ce sont surtout les verts et les libéraux qui font l'effrai de ce scrutin: ils disparaissent de la scène politique du land / de Sachse Anhalt. Quant aux ex-communistes du PDS avec près de vingt pourcent, ils occupent la troisième place sur l'échiquier politique et se présentent / en arbitre pour entrer dans un gouvernement. Un gouvernement que les vainqueurs, les socialistes du SP $D$, ne peuvent constituer seuls - le SP $D$ vient d'ailleurs d'annoncer qu'il est prêt d'entamer des discussions avec les chrétiens démocrates. A quelques mois des élections législatives, une coalition $\mathrm{SP} D-\mathrm{CDU} /$ paraît difficile à mettre sur pied. Quant à une alliance entre les anciens communistes et les socialistes, elle paraît improbable, bref, pour l'SP $D$, cette victoire ne sera pas facile à gérer.

Cercle des arts [8/1 1998]

Animateur: Frédéric MITTERRAND (FM)

Invités: Claude PÉTRI (CP), Viviane FORESTER (VF), Alain de BOTTON (AB), Pierre ASSOULINE (PA), Jean-Yves TADIÉ (JYT), Stéphane BERNE (SB), Pierre LE TANNE (PL)

(FM-1) Eh bien, Alain Botton, ce qui me fascine, c'est que, venant // bien plus tard, à un autre moment, à dix-huit ans vous découvrez Proust, avec la même allégresse et la même gravité. Comment ça s'est passé? A Londres?

(AB-1) Ça m'a peut-être aidé que j'ai découvert Proust à Londres, parce que... je ne sais pas, les Français sont toujours en train de / relire Proust, on ne trouve jamais un Français qui est en train de lire Proust pour la première fois. Donc il y a un genre de snobisme proustien en France et je crois que ça c'est sans doute un problème pour les Français, ils ont un problème d'appréciation, parce que, d'avoir trop de respect pour un livre, ça n'aide jamais. Et donc j'ai eu cette liberté que j'ai découvert Proust, enfin, les Anglais ont beaucoup de défauts, ils ne savent pas faire la cuisine, mais grand avantage, ils ne s'intéressent pas trop à la culture. Et donc ça laisse une certaine... ça 
permet une allégresse, une légèreté. Et donc / quand j'ai découvert Proust, à dix-huit ans, j'ai découvert ça... pas dans un cadre universitaire, simplement, pour moi-même.

\section{Analyse prosodique du corpus}

En tout, l'analyse observe 3131 unités accentuelles comportant un accent final délimitatif. Tous les autres types accentuels (résultant évidemment de l'accent d'insistance lexémique) sont représentés à 1159 reprises. Cela signifie en gros que 31,7\% d'unités accentuelles contiennent une syllabe accentuée non-finale. Dans la plupart des cas, cet accent marque la première syllabe de l'unité. L'analyse qui suit se donne pour objectif principal de démontrer quelle est la nature et quelles sont les fonctions des accents qui frappent les syllabes autres que la dernière.

\subsection{L'accent d'insistance}

La distinction spatiale et distributive entre l'accent final et l'accent d'insistance à l'intérieur de l'unité accentuelle (intonative) actualisée évite généralement toute ambiguïté communicative. L'accent final en rapport synchronisé avec l'intonème se manifeste comme une orientation énonciative qui termine l'unité accentuelle; selon les principes de contraste prosodique, l'accent d'insistance est toujours placé sur une syllabe non-finale, le plus souvent la première ou la deuxième. ${ }^{16}$ La situation est moins claire dans le cas des unités accentuelles monosyllabiques, (non)marquées ${ }^{17}$ d'ailleurs le plus souvent par un accent final délimitatif. Dans ces cas-là, il est éventuellement possible de reconnaître l'accent d'insistance au moyen de certaines de ses caractéristiques substantielles.

L'accent d'insistance est d'ailleurs un procédé prosodique dont la substance rappelle essentiellement la production d'un accent de n'importe quel type. Il est donc également le résultat d'un investissement relativement augmenté de l'énergie expiratoire par le locuteur. Mais il se distingue assez nettement des réalisations accentuelles finales en quelques points articulatoires et distributifs:

\footnotetext{
${ }^{16}$ L'accent d'insistance affecte régulièrement la deuxième syllabe du lexème exposé quand celui-ci commence par une syllabe ouverte ne comportant que le noyau vocalique. Par exemple: dans la phrase C'est époustouflant, c'est la deuxième syllabe qui reçoit l'accent d'insistance si le locuteur choisit de marquer prosodiquement l'adjectif selon l'expression de son attitude (émotive) envers ce qui est qualifié par le lexème en question.

${ }^{17}$ Etant donné la prévisibilité positionnelle de l'accent final et son appartenance à la structure inhérente du code linguistique français, il est difficile de dire sans réserve que la dernière syllabe de l'unité accentuelle, en français, soit marquée par ce fait prosodique. De par leur valeur contrastive (donc négative ou non-oppositionnelle du point de vue phonologique), les manifestations de l'accent délimitatif ne s'accordent pas à la définition «verticale» du marquage; cette définition pose qu'une unité linguistique est marquée lorsqu'elle possède une particularité structurale qui l'oppose aux autres unités de même nature de la même langue. L'accent final français, comme nous le savons, n'entre pas dans les rapports paradigmatiques de binarité, puisque le contraste accentuel opère sur l'axe horizontal (syntagmatique) de la chaîne parlée.
} 
- sa réalisation vocalique s'accompagne le plus souvent d'une intensification de la consonne dans les syllabes à structure $\mathrm{CV}$; dans ce cas, la consonne (surtout les occlusives et les nasales) peut également se trouver relativement allongée;

- si la syllabe affectée par l'accent d'insistance commence par une voyelle, l'articulation vocalique intensifiée est souvent précédée par une occlusion glottale ou par une aspiration pharyngale;

- parmi les moyens prosodiques servant à réaliser une insistance, l'emploi de la pause intervient fréquemment devant la syllabe affectée; le rôle de la pause est ici de distinguer perceptiblement et fonctionnellement la syllabe affectée par l'accent d'insistance et la syllabe (in)accentuée précédente.

Ces instruments organisateurs de la chaîne parlée ne sont jamais employés par le locuteur lorsqu'on réalise un accent final. Dans le continuum transitoire de la parole, celui-ci se trouve toujours (sauf le cas des unités accentuelles monosyllabiques) physiquement lié à la syllabe inaccentuée précédente.

Nous avons constaté dans l'analyse de l'accentuation, propre au discours médiatique des animateurs et journalistes (cf. pourcentage des insistances par unité accentuelle), que l'accent d'insistance représente l'un des procédés prosodiques les plus fréquents dans la production du discours médiatique. Il paraît évident que l'usage de l'accent d'insistance s'inscrit dans l'ensemble des stratégies énonciatives des locuteurs dont l'intention communicative est d'attirer un maximum d'attention de la part du spectateur ${ }^{18}$ et d'intégrer l'intelligibilité du message. Etant donné sa fonction dans l'organisation de la chaîne parlée, l'accent d'insistance se prête avec efficacité à la volonté du locuteur soit d'exprimer une attitude envers le contenu du message (étonnement, horreur, ironie...) soit de distinguer logiquement la signification (et le sens) de deux lexèmes dans un énoncé. Dans l'exemple (énoncé par un interviewé, donc non par un locuteur professionnel) «...les nouveaux francs, il faut que je les convertisse en anciens francs, alors je ne vous dis pas quand ça sera l'euro», c'est l'accent d'insistance sur les deux adjectifs qui soutient la différenciation des significations.

Il s'agit là d'un exemple qui confirme la description habituelle du fonctionnement de l'insistance en français. La fonction logique de cet accent n'opère donc pas moins

18 Nous comprenons le rôle du spectateur dans ce type spécifique de communication comme une participation «passivisée» à la relation entre un locuteur (professionnel) et un «interlocuteur» qui en fait $n$ 'en pas un puisqu'il n'a normalement aucune ingérence directe au processus communicatif. La «communication» médiatique doit effectivement être comprise comme une communication unilatérale (et donc tronquée) où il n'y a au moment de la parole qu' un seul locuteur et un nombre considérable d' «interlocuteurs» (nombre correspondant au nombre respectif des spectateurs). C'est justement ce type de communication que rend possible la technologie médiatique, multipliant momentanément un message linguistique par le nombre de récepteurs. L'impossibilité du spectateur d'intervenir directement à l'échange des messages énoncés (donc sa passivité) ouvre d'autant plus les possibilités d'influence que peut exercer un locuteur actif sur la formation des compétences linguistiques en se servant du code, utilisé par le média. 
dans le discours médiatique que dans les discours «spontanés» des locuteurs non-professionnels. On verra plus loin que la routine de l'accentuation insistante dans le discours médiatique élargit son champ d'opération et que l'on doit ajouter quelques précisions importantes - quant au comportement des syllabes accentuées non-finales - à ce qui est normalement dit des schémas accentuels français.

\section{Effets de l'insistance dans l'accentuation du discours médiatique}

L'analyse perceptive du corpus des textes médiatiques a montré que, en général, l'organisation syntaxique des énoncés ne conditionne pas nécessairement les procédés qui déterminent prosodiquement les différentes parties de la chaîne parlée française. Il paraît au contraire que la stratégie accentuelle dépend plus de la situation communicative et des critères phonétiques dans la formation du discours médiatique. L'examen accentuel des exemples essaiera en principe de montrer l'indépendance des structures prosodiques par rapport à l'organisation syntaxique de la phrase. On montrera que le fonctionnement de l'insistance dans le discours médiatique est un point de départ pertinent pour une réflexion sur la nature de l'unité accentuelle française et sur le dynamisme des fonctions accentuelles en français.

\subsection{Critères syntaxiques}

La nature virtuelle de l'accent final peut généralement être démontrée par l'actualisation des unités accentuelles relativement longues. Ce type de formation des unités accentuelles (et de rythmisation du discours) est surtout caractéristique pour des énonciations «spontanées» à débit rapide.

«La finale a été symptomatique // trois cent cinquante francs un billet de finale // alors qu'aux Etats-Unis, plus de mille francs.»

On observe facilement que la plus longue unité accentuelle (celle d'entre les deux paires de barres obliques, réalisée donc entre deux pauses relativement longues) contient deux unités minimales virtuelles, déterminables par des moyens syntaxiques, mais qui se trouvent réunies en une seule, et cela en conséquence d'un tempo accéléré de l'énonciation.

L'exemple suivant introduit dans l'unité accentuelle étendue l'usage de l'accent d'insistance.

«... le premier ministre Chrétien a abordé la question des droits de la personne avec le président Fidel Castro // dès qu'il s'est retrouvé seul avec lui // en demandant la libération de quatre prisonniers / d'opinion.» 
L'unité accentuelle actualisée «dès qu'il s'est retrouvé seul avec lui» contient également au moins deux unités virtuelles minimales, réunies par un seul accent final. Mais la participation des deux accents d'insistance révèle quelques particularités essentielles du schéma accentuel dans le discours médiatique. La forme verbale ${ }^{19}$ qui termine la première unité minimale (déterminée syntaxiquement) se trouve désaccentuée, parce que l'énergie expiratoire, nécessaire à la production de l'accent, est dépensée postérieurement pour l'insistance sur le lexème central de l'unité entière. Le contraste acoustique entre la syllabe affectée par l'accent d'insistance et son entourage est nécessairement plus exprimé que celui entre la finale et la précédente inaccentuée.

De plus, l'insistance sur la conjonction «dès que» démontre l'expansion fonctionnelle de l'insistance: du lexème, son opération se répand sur toute une unité accentuelle minimale, bien que celle-ci risque de ne pas s'actualiser réellement. Sa position lui attribue le statut de délimitateur d'une partie prosodiquement marquée à la frontière antérieure. Davantage: l'accent d'insistance affecte ici la première syllabe non pas d'un lexème, mais d'une unité syntaxique. Cette première syllabe fait partie d'une conjonction qui, selon l'acception traditionnelle de l'accentuation en français, n'est généralement pas accentogène.

\subsection{Accentuation des proclytiques déictiques}

L'usage fréquent de l'accent d'insistance dans le discours médiatique (et plus particulièrement dans celui des présentateurs et des journalistes), à l'origine, est le résultat d'une tendance prononcée de marquer stratégiquement les lexèmes qui forment le centre informatif de l'énoncé ou de ses parties. Mais ce procédé accentuel a progressivement pris de l'envergure même dans les cas où la première syllabe du lexème (ou du constituant syntaxique) n'est pas accentogène. C'est le rôle délimitatif de l'insistance médiatique, positionnée en effet au début de l'unité, qui est devenu plus important que sa capacité de focalisation informatrice. On proposera maintenant l'exemple qui rend compte à la fois de l'actualisation des unités accentuelles minimales (dans une énonciation «saccadée» dans l'intention d'attirer l'intérêt du destinataire par la focali-

${ }^{19}$ La divergence des critères accentuels par rapport à la structuration sytaxique de la phrase est encore affirmée si l'on observe le comportement accentuel sur les verbes. La grande plupart des théories syntaxiques posent le verbe en position centrale de l'organisation de la phrase, lui attribuant le rôle régisseur par rapport à tous les autres constituants immédiats (principe de dépendance, cf. le stemmas de Tesnière). Si le centre syntaxique d'un message linguistique est donc occupé par l'actualisation de la forme verbale, celle-ci n'est pas centralisée dans les schémas accentuels. D'abord, la forme verbale se trouve régulièrement au terme de l'une des unités accentuelles virtuelles dont l'actualisation dépend des facteurs communicatifs, stratégiques et phonétiques. Un verbe se trouve donc facilement désaccentué si le débit de la parole est rapide ou s'il se trouve en proximité directe d'un accent d'insistance qui lui «reprend» le trait accentuel. Contrairement à cela, l'adverbe et le numéral, en tant qu'éléments de grande valeur sémantique spécifique, sont beaucoup plus susceptibles non seulement de recevoir un accent final, mais d'ètre marqués par l'accent d'insistance qui a pour but de focaliser leur signification et leur valeur informative. 
sation des fragments informatifs du message) et l'emploi de l'insistance sur les syllabes (ou mot grammaticaux), normalement non-accentogènes.

«Dimanche noir, catastrophe, signal d'alarme, ce matin, les journaux allemands rivalisent de titres choc.»

La formation du schéma accentuel dans cette actualisation (c'est Jean-Paul Gérouard, un journaliste, qui parle) est un exemple typique des tendances prosodiques générales dans le discours médiatique français. Les trois premières unités commencent par des lexèmes qui ont une pleine valeur sémantique; dans ces cas-là, on peut donc parler de la focalisation du sens telle qu'elle est décrite par les principes généraux de l'insistance. Mais la continuation ne va plus par ce même chemin structurel. La première syllabe dans les unités «ce matin» et «les journaux allemands» est, syntaxiquement parlant, porteuse de déterminants déictiques (indice démonstratif et article défini). Selon les règles de l'accentuation et de l'accentuabilité, ces deux syllabes ne pourraient donc aucunement recevoir de marques accentuelles. Le locuteur n'est toutefois pas empêché de réaliser l'insistance sur les deux premières syllabes en question. L'influence de l'usage systématique des accents d'insistance (qui ont, à l'origine, une valeur uniquement logique ou émotive), dépasse donc les cadres traditionnels de la description accentuelle, parce que la stratégie énonciative des locuteurs médiatiques utilise, sans égard à la normativité accentuelle explicite, l'instrument de l'insistance sur les proclytiques.

\subsection{Un critère phonétique: le contraste}

Dans l'introduction, traitant des généralités accentuelles, nous avons indiqué le contraste matériel et fonctionnel que forme la présence d'un accent avec son contexte phonique, autrement dit, avec les syllabes dites inaccentuées. L'accent final français, nous l'avons également dit, se réalise dans une projection acoustique complexe de l'effort expiratoire, combinée à l'événement intonatif. De nombreuses analyses antérieures $^{20}$ à celle-ci ont montré que la dernière syllabe d'une unité rythmique, c'est-à-dire la syllabe sur laquelle s'opère la conclusion intonative, est précédée d'une autre syllabe, prosodiquement moins expressive, mais pas pour autant moins fonctionnelle dans le contraste, dont les valeurs acoustiques sont directement opposées à la prosodie du terme de l'unité. Cela signifie, en d'autres termes, que la conclusion intonative de continuation ou d'interrogation (donc montante) sera nécessairement précédée d'un baissement da la hauteur tonale sur l'avant dernière; contrairement à cela, un événement intonatif désignant la finalité (donc descendant) est introduit par une syllabe relativement plus haute. Le contraste de l'accent final s'effectue donc surtout en substance de

20 cf. Vitez (1995a).

116 
la variation intonative. Il s'agit maintenant de voir, quelles sont les qualités et les quantités contrastives qui font fonctionner l'accent d'insistance et les accents qui en dérivent dans le discours médiatique français.

L'orientation générale dans la structuration des schémas accentuels, propres aux langues à accent fixe, prévoit un nombre suffisant de syllabes inaccentuées qui s'interposent entre deux syllabes accentuées. Une telle définition distributive des syllabes prosodiquement marquées demande une précision dans le cas de l'unité accentuelle française. Le fait que les procédés prosodiques se servent en même temps de l'accent final et de l'accent d'insistance (lexémique ou syntagmatique) dans une même unité, a pour conséquence inévitable le contact direct entre la fin d'une unité (accent final, intonation) et le début de la suivante (insistance).

\section{«Disons qu'ils avaient une situation / conflictuelle, tendue...»}

L'exemple est pris d'une réplique, énoncée par une invitée au débat télévisé où le registre discursif général était caractérisé d'un niveau hautement intellectuel. Ce niveau énonciatif se distingue par un emploi relativement fréquent des accents d'insistance logique, semblable à celui des présentateurs des journaux de télévision. La distinction fonctionnelle entre les accents de type final et celui d'insistance serait impossible dans les unités «conflictuelle» et «tendue» si les deux accents étaient réalisés par le recours à la même combinaison de paramètres acoustiques. Nous savons déjà que l'accent final est réalisé en interaction avec l'intonation; sa substance contrastive sera donc la variation de la hauteur tonale. Quand l'accent d'insistance se trouve dans une telle proximité de l'accent final (ou le suit ou précède directement), il faut bien que sa substance (ou ses propriétés) soient différentes. Il y a ici trois explications possibles. Premièrement, comme on l'a indiqué plus haut pour les syllabes directement antéposés à l'événement intonatif, la courbe intonative pourrait prendre sur cette syllabe la direction opposée par rapport à l'accent final. Deuxièmement, ce qui à première vue paraît plus plausible, l'accent d'insistance se réaliserait au moyen d'une intensité relativement augmentée, donc d'une autre substance acoustique dont l'emploi exprime bien plus fortement la différence impressive entre les deux syllabes. La troisième variante réconcilierait les deux premières en avançant que l'insistance se réalise en combinant toutes les deux substances acoustiques ou, autrement dit, tous les deux procédés phonétiques. Cette troisième possibilité serait peut-être la plus proche de la réalité, surtout parce que la constante réalisation intonative et accentuelle de la dernière syllabe de l'unité indique un certain automatisme dans le processus de l'énonciation. En plus, ce fait nous amène à croire que l'accent d'insistance (au moins dans sa valeur focalisatrice sémantique) est une conséquence du choix stratégique conscient, effectué par le locuteur dans l'intention de passer un message linguistique cohérent. 


\subsection{La pause}

L'utilisation de la pause s'inscrit valablement dans les procédés stratégiques qui s'engagent à distinguer les accents entre eux. En même temps, on peut constater que la pause accompagne surtout les actualisations de l'accent d'insistance. Claire Blanche-Benveniste ${ }^{21}$ postule en outre que l'accent final peut être suivi par une pause, mais ne peut pas en être précédé; contrairement à cela, il est caractéristique de l'accent d'insistance que les locuteurs le réalisent par suite d'une pause. Il faut toutefois ajouter à cela une précision: l'accent final peut être précédé d'une pause, mais à condition qu'il frappe l'unique syllabe de l'unité rythmique, que son unité soit donc monosyllabique et autonome. Ces situations extraordinaires et cette distribution de pauses peuvent être observées dans un certain type de discours où le locuteur parle par fragments et lentement. Ce type de l'énonciation est le plus souvent utilisé par les locuteurs (présentateurs, politiciens, professeurs...) dont l'intention est de clairement poser leur message pour qu'il soit compréhensible à toutes les sensibilités intellectuelles présentes dans la communication.

\section{Conclusions}

Les deux questions essentielles, posées par les résultats de cette analyse, concernent les fonctions des deux procédés fondamentaux, issus des caractéristiques de l'accentuation françaises. Toutes les deux sont relatives à la nature et à l'extension de l'unité accentuelle; plus particulièrement, le première problématise le fonctionnement et la réalisation de l'accent final, l'autre affecte l'usage "automatisé» de l'insistance et ses conséquences systématiques dans le discours médiatique.

\subsection{La double délimitation de l'unité accentuelle}

L'affaiblissement de la fonction fondamentale de l'accent d'insistance, notamment celle de focaliser la valeur sémantique d'un lexème selon l'attitude émotive ou logique du locuteur, et son transfert aux unités non-lexémiques, démontrent clairement une tendance systématique: les insistances syntagmatiques occupent leur position sur la première syllabe de l'unité accentuelle, et cela sans égard à la valeur sémantique de l'unité affectée. Utilisés automatiquement dans le discours médiatique, les accents d'insistance sont sémantiquement démotivés, leur position est prévisible. Les accents d'insistance syntagmatiques sont donc en parfaite analogie avec les propriétés mécanisées de l'accent final dont la fonction est réduite à la délimitation de la fin des unités

21 Blanche-Benveniste (1991), p. 165: «[...] la distinction est donc fondée sur des critères distributionnels; l'accent final accepte les tons dynamiques (le ton montant et le ton descendant), la syllabe peut être allongée et suivie d'une pause. Cela n'est pas le cas de l'accent d'insistance. L'allongement de la syllabe [...] et l'insertion d'une pause après celle-ci sont exclus; en revanche, l'insertion d'une pause devant l'accent d'insistance ne pose aucun problème alors qu'elle serait ressentie comme un raté si elle se trouvait devant un accent final.». 
accentuelles. Il paraît donc que l'insistance syntagmatique, dépourvue de valeur focalisatrice, est en fonction de marquer la limite de la même unité accentuelle, mais sur la première syllabe. Comme l'accent final, elle sert de point de repère prosodique au destinataire du message.

\subsection{La fonction phatique de l'insistance}

Les conséquences de la tendance routinière, fortement exprimée dans le discours médiatique, à utiliser l'insistance syntagmatique, révèlent donc une modification déterminante de la fonction de ce type d'accent. Ses propriétés contrastives, comme nous l'avons vu, opèrent en tant qu'instrument de l'orientation sur la première syllabe de l'unité. La fréquence de l'insistance non-lexémique affirme en même temps la valeur phrastique de l'accent final. Mais la fonctionnalité de l'accent final (délimitation) remet en cause la fonction orientative de l'insistance sur la première syllabe. $\mathrm{Si}$ l'on considère la redondance fonctionnelle dans la langue en tant qu'opération cohérente de structures énonciatives autonomes, on verra dans le marquage accentuel du début de l'unité rythmique un moyen prosodique qui, à l'intérieur du message linguistique, est concentré sur son fonctionnement phatique. Cela signifie que l'accent d'insistance syntagmatique, dépourvu de motivation sémantique, fonctionne comme une information automatisée, produite par le locuteur qui assure par là sa propre présence, la présence de son acte de parole et la présence de son intention communicative fondamentale.

\section{Bibliographie}

AUBERGÉ, Véronique (1991): La synthèse de la parole. Des règles aux lexiques, thèse de doctorat, ICP-INPG/Université Stendhal - Grenoble III et CRISS/ Université Pierre Mendès France - Grenoble II.

BLANCHE-BENVENISTE, Claire (1991): Le français parlé. Études grammaticales, coll. «Sciences du langage», Éditions du CNRS, Paris.

CREISSELS, Denis (1993): Les notions primitives de la syntaxe, in: Vers une rénovation de la grammaire et de sa terminologie, LIDIL 8, Presses Universitaires de Grenoble, pp. 51-83.

Delattre, Pierre (1966): Studies in French and Comparative Phonetics, Mouton, London-Haag, pp. 65-68 (1938).

DelaTTRE, Pierre (1966a): Les dix intonations de base du français, in: French Review 40/1, pp. 1-14.

DelatTRE, Pierre (1966-1967): The Distinctive Function of Intonation, in: The General Phonetic Characteristics of Languages, University of California, Santa Barbara, pp. 81-102. 
DeLL, François (1982): L'accentuation dans les phrases en français, in: Les représentations en phonologie (éd. F. Dell, D. Hirst et J. R. Vergnaud), Hermann, Paris.

FóNAGY, Ivan (1969): Accent et intonation dans la parole chuchotée, in: Phonetica 20, pp. 177-192.

FóNAGY, Ivan, LÉON, Pierre (1979): L'accent en français contemporain, Studia Phonetica 15, Didier, Ottawa.

FóNAGY, Ivan (1979a): L'accent français: accent probabilitaire. Dynamique d'un changement prosodique, in: Studia Phonetica 15, Didier, Ottawa, pp. 123-233.

FóNAGY, Ivan (1979b): Fonction prédictive de l'intonation, Studia Phonetica 18,

Didier, Ottawa, pp. 113-120.

FóNAGY, Ivan (1983): La vive voix. Essais de psycho-phonétique, Payot, Paris.

GARDE, Paul (1968): L'accent, Presses Universitaires Françaises, Paris.

HALLIDAY, M.A.K. (1989): Spoken and written language, 1ère éd. 1985, Oxford University Press.

JAKOBSON, Roman (1976): Six leçons sur le son et le sens, Éditions de Minuit, Paris.

LEVELT, Willem J. M. (1995): Speaking. From Intention to Articulation, The Massachusetts Institute of Technology Press, Cambridge, Massachusetts.

LuCCI, Vincent (1983): Etude phonétique du français contemporain à travers la variation situationnelle, Publications de l'Université des langues et des lettres de Grenoble.

MARTIN, Philippe (1980): Une théorie syntaxique de l'accentuation en français, in: Studia Phonetica 15, Didier, Ottawa, pp. 1-12.

Rossı, Mario (1979): Le français, langue sans accent?, in: L'accent en français contemporain, Studia Phonetica 15, Didier, Ottawa, pp. 13-51.

RossI, Mario, DI CRISTO, Albert, HIRST, Daniel, MARTIN, Philippe, NISHINUMA, Yukihiro (1981): L'intonation. De l'acoustique à la sémantique, Klincksieck, Paris.

VITEZ, Primož, AUBERGÉ, Véronique (1995): The Intonation Gesture of Slovene: first indications, in: Proceedings of 4th European Conference on Speech Communication and Technology, tome 3, Madrid, pp. 2073-2075.

VITEZ, Primož (1995a): Protistavna analiza francoske in slovenske stavčne intonacije, thèse de 3 e cycle, Faculté des lettres, Université de Ljubljana.

VITEZ, Primož (1995b): Analyse contrastive de l'intonation de la phrase française et slovène, in: Linguistica XXXV/2, Ljubljana, pp. 257-274.

VITEZ, Primož (1997): Accent d'intensité et action intonative en français moderne, in: Linguistica XXXVII, Ljubljana, str. 71-80.

VITEZ, Primož (1999): Od idealnih jezikovnih struktur $k$ strategiji realnega govora, in: Slavistična revija 1, pp. 23-48, Ljubljana.

WALTER, Henriette (1976): La dynamique des phonèmes dans le lexique français contemporain, France Expansion, Paris. 
Povzetek

\section{NAGLASNA DINAMIKA FRANCOSKEGA MEDIJSKEGA GOVORA}

Naglasoslovni sklepi in splošnejša jezikoslovna interpretacija s korpusom izpričanih prozodičnih pojavov se hkrati opirajo na izsledke podrobne zaznavne analize medijskih besedil in na tradicionalni opis naglasnega sistema $v$ francoščini. Razlikovanje je nujno potrebno zaradi razčlembe in povzemanja naglasnih posebnosti $\mathrm{v}$ medijskem govoru, ki predstavljajo bistvene prvine odstopanja realne govorne pojavnosti od idealno formuliranih lastnosti normativnega upovedovanja. Frekventna pojavnost teh odstopanj $v$ specifičnem komunikacijskem položaju je obenem $v$ tematski srži pričujočega prispevka in disertacije $z$ naslovom Premiki v naglasnem sistemu francoskega medijskega govora, ki jo članek povzema.

$\mathrm{V}$ ospredje razmisleka je ves čas postavljeno vprašanje funkcionalnosti dveh temeljnih postopkov, ki izhajata iz značilnosti francoskega naglasnega sistema. Oba sta hkrati povezana s problemom obsega in definicije naglasne enote, vendar prvi posebej zadeva delovanje in realizacijo končnega zamejitvenega naglasa, drugi pa uporabo poudarjanja in sistemske posledice te uporabe $\mathrm{v}$ procesu upovedovanja.

Položajna prekrivnost končnega zamejitvenega naglasa $\mathrm{z}$ realizacijo intonema spodbuja $\mathrm{k}$ sklepu, da $\vee$ francoščini tako naglasni kot intonacijski postopki sodijo med stavčnofonetična sredstva, ki povečini zadevajo razmeroma obsežne dele aktualizirane govorne verige. S tem spoznanjem se vprašanje funkcionalnosti odpira le $v$ manjši meri; bolj aktualen je ponoven razmislek o realizacijski substanci zamejitvenega naglasa in s tem $v$ zvezi problem veljavnosti splošno sprejete terminologije, $\mathrm{ki}$ se naslanja na značilnosti snovne izvedbe določenih prozodičnih sredstev.

Za leksemsko poudarjanje je značilno, da je njegova zavestna uporaba kot govornega strateškega sredstva - s stališča koherentnosti sporočanja - posledica govorčeve namerjenosti $\mathrm{k}$ notranji enotnosti in nedvoumnosti upovedovanja. Analiza vpliva frekventne rabe leksemskega poudarjanja $v$ medijskem govoru in nekaterih drugih tipih govornih izvedb je $v$ obravnavanem korpusu pokazala, da v takšnem (rutinskem) nizanju naglasnih shem obstaja močna tendenca prehajanja poudarkov na prve zloge naglasnih enot, kjer ne le ne zadevajo leksemov, temveč tudi ne osmišljujejo specifične pomenske vrednosti, ki jo leksemsko poudarjanje po definiciji izpostavlja. V korpusu obdelano poudarjanje naslonk $v$ položaju začetnega zloga sintagmatsko oblikovanih naglasnih enot gre interpretirati prav kot posledico poudarnega mehanizma, ki s samodejnim delovanjem znotraj določenih upovedovalnih okoliščin preneha središčiti pomen enote, ki jo zadeva. Takšni poudarki zlasti pri medijskih govorcih postajajo sistematični del naglasnih shem in jih ne moremo več opredeliti kot leksemske. Polje njihovega delovanja niso naslonke, ki jih zaznamujejo, temveč celotne naglasne enote. Prozodični postopek, ki takšne poudarke udejanja, zato imenujemo sintagmatsko poudarjanje.

To pojavnost je možno interpretirati kot naglasno sredstvo, ki deluje kot zamejevalec prozodično določenih enot na prvem zlogu. Prepoznavna pogostnost in kontrastnost sintagmatskega poudarka $v$ takšnem položaju znotraj neleksemskih naglasnih enot govorita v prid tezi o stavčni vrednosti končnega naglaševanja. Vendar se glede na dejstvo, da zamejitveno funkcijo že opravlja končni dinamični naglas, ob delovanju sintagmatskega poudarka zastavlja še vprašanje redundantnosti začetnega zamejevanja enote. Če redundanco $v$ jeziku pojmujemo kot koherenčno delovanje avtonomnih struktur, potem gre $v$ poudarnem zaznamovanju videti prozodično sredstvo, ki znotraj govornega sporočila poleg zamejitvene opravlja tudi fatično jezikovno funkcijo. To pomeni, da semantično nemotivirani sintagmatski naglas deluje kot govorčeva avtomatično tvorjena informacija naslovniku o fizični prisotnosti njegovega lastnega govornega dejanja in s tem tudi o prisotnosti njegovega osnovnega sporočanjskega namena. 